\title{
INFLUÊNCIA DO TAMANHO E DA RIGIDEZ DOS OBJETOS NOS AJUSTES PROXIMAIS E DISTAIS DO ALCANCE DE LACTENTES
}

\author{
Rocha NACF, SiLVA FPS E Tudella E \\ Departamento de Fisioterapia, Setor de Fisioterapia em Neuropediatria, Universidade Federal de São Carlos, São \\ Carlos, SP - Brasil \\ Correspondência para: Nelci Adriana Cicuto Ferreira Rocha, Rua Miguel Mazzei, 130, Residencial Samambaia, CEP \\ 13565-570, São Carlos, SP - Brasil, e-mail: acicuto@power.ufscar.br
}

Recebido: 26/10/2005 - Aceito: 21/12/2005

\begin{abstract}
RESUMO
Contextualização: Estudos têm identificado que as propriedades dos objetos induzem os ajustes no alcance; no entanto, poucos investigaram a influência específica do tamanho e rigidez dos objetos em lactentes jovens. Objetivo: Verificar se lactentes de 4 a 6 meses realizam ajustes proximais e distais ao alcançarem objetos de diferentes tamanhos e rigidez. Métodos: Nove lactentes saudáveis foram posicionados em uma cadeira inclinada a $50^{\circ}$. Quatro objetos foram apresentados, um rígido grande (RG), um rígido pequeno (RP), um maleável grande (MG) e um maleável pequeno (MP), por um período de 1 minuto cada. Em um total de 384 alcances, foram analisados os ajustes proximais (alcance uni e bimanual) e distais (orientação da mão horizontal, vertical e oblíqua; mão aberta, fechada e semi-aberta) e o sucesso do alcance dos objetos. Resultados: Constatou-se ajuste bimanual para o objeto RG e unimanual para os demais. A orientação da mão oblíqua foi predominante no toque dos objetos, enquanto para a preensão dos mesmos, a predominância foi a vertical, principalmente para o objeto RG. A orientação horizontal não foi observada na preensão do objeto RG. A mão semi-aberta foi mais freqüente no início do alcance para todos os objetos, enquanto no toque do objeto RG a mão aberta foi predominante. O sucesso do alcance foi maior para os objetos maleáveis (MG, MP) do que para os rígidos (RG e RP). Conclusão: Lactentes jovens estudados são capazes de planejar e ajustar seus movimentos baseados na percepção das propriedades físicas dos objetos, o que sugere interação percepção-ação.
\end{abstract}

Palavras-chave: Propriedades físicas dos objetos, alcance, preensão, percepção, lactente, affordances.

\section{ABSTRACT \\ Influence of Object Size and Rigidity on Proximal and Distal Adjustments to Infant Reaching}

Background: Studies have identified that object properties lead to adjustments to reaching. However, few have investigated the specific influence of object size and rigidity among young infants. Objective: To verify whether four to six-month-old infants make proximal and distal adjustments when reaching for objects of different sizes and rigidity. Method: Nine healthy infants were seated on a chair inclined at $50^{\circ}$. Four objects were presented to them: one large rigid (LR), one small rigid (SR), one large malleable (LM) and one small malleable object (SM), each for a one-minute period. A total of 384 reaches were analyzed to verify proximal adjustments (single-hand and two-hand reaching) and distal adjustments (horizontal, vertical and oblique hand orientation; opened, closed and half-open hand), and the success in reaching the objects. Results: The infants exhibited twohand adjustments for the LR object and single-hand adjustments for the other objects. Oblique orientation was predominant for touching the objects, while vertical orientation was predominant for grasping them, particularly the LR object. Horizontal orientation was not observed for grasping the LR object. At the start of reaching for all objects, the hands were most frequently half-open, while the hands were predominantly open when touching the LR object. Success was greater when reaching for malleable objects (LM, SM) than for rigid objects (LR and SR). Conclusion: The young infants studied were capable of planning and adjusting their movements on the basis of their perceptions of the physical properties of the objects, which suggests that perception-action interaction was occurring.

Key words: physical properties of objects, reaching, grasping, perception, infant, affordances. 


\section{INTRODUÇÃO}

A aquisição da habilidade de alcançar e apreender objetos constitui um importante marco no desenvolvimento motor e cognitivo no primeiro ano de vida dos lactentes ${ }^{1,2}$. Aprender a coordenar e ajustar os movimentos dos membros superiores para alcançar e apreender objetos é, portanto, um processo essencial para que o lactente aprenda sobre o ambiente e atinja seus objetivos com maior precisão².

Para que os objetos sejam alcançados e apreendidos com precisão, deverão ocorrer dois tipos de ajustes de movimentos dos membros superiores: o ajuste proximal, considerado como a iniciativa de direcionar um ou ambos os membros superiores (alcances uni e bimanual) ao alvo apresentado; e o ajuste distal, que se refere ao posicionamento da mão e dos dedos para fazer contato e apreender o objeto ${ }^{3}$. Alguns pesquisadores relatam que os ajustes proximais bimanuais iniciam por volta dos 5-6 meses ${ }^{3,4}$ e os ajustes distais, por volta dos 7-9 meses ${ }^{5}$. Outros pesquisadores, ainda verificaram que os ajustes proximais e distais são guiados pela informação disponibilizada pelas propriedades físicas dos objetos ${ }^{6,7,8}$.

Jeannerod ${ }^{9}$ sugere que as propriedades físicas intrínsecas dos objetos, tais como tamanho, forma, textura e peso, afetam o posicionamento das mãos e dedos (ajustes distais) em relação ao objeto, enquanto as propriedades extrínsecas, tais como distância, localização e orientação do objeto, influenciam a trajetória de braço e mão (ajustes proximais) em direção ao objeto.

Estudos têm constatado que o tamanho e a rigidez dos objetos influenciam nos ajustes manuais em lactentes apenas por volta dos 8-9 meses de idade ${ }^{10,3}$. Segundo Corbetta, Thelen e Johnson ${ }^{10}$, o tamanho do objeto influenciará a estratégia do lactente em usar uma ou duas mãos para tocar e apreender objetos, ou seja, uma mão para os objetos pequenos e duas para os grandes, sendo que o objeto maleável oferece a opção de usar apenas uma mão para alcançar e apreender os objetos independentemente de seu tamanho. Contudo, o efeito do tamanho e da rigidez nos ajustes distais do alcance (orientação e abertura da mão) não foi verificado em tal estudo. Referente aos ajustes distais, Fagard ${ }^{3}$ verificou aumento da abertura da mão de acordo com o tamanho do objeto, diminuição da orientação horizontal da mão com o aumento da idade, bem como aumento na orientação vertical ao tocar o objeto. Fagard ${ }^{3}$, no entanto, não verificou a relação da orientação das mãos com o tamanho do objeto.

Dessa forma, nota-se que, embora a literatura evidencie o desenvolvimento do alcance e relate o fato de que as propriedades físicas dos objetos induzem ajustes proximais e distais de movimentos, há poucos estudos que investigam o aprimoramento de ambos considerando as influências específicas das propriedades de tamanho e rigidez dos objetos em lactentes jovens de 4 a 6 meses de vida.
Além disso, observa-se que há um consenso de que os lactentes possuem sistemas perceptuais ativos que captam as informações específicas do ambiente ${ }^{11,10}$ e de que, nesse período, o sistema exploratório manual (visual e motor) desenvolve-se, tornando possível a percepção das affordances, que se trata da relação entre o que o ambiente oferece como possibilidade de ação e as capacidades do organismo ${ }^{12}$. Dessa maneira, as características distintas dos objetos fornecem informações que direcionam ações específicas ${ }^{13}$. Atualmente pesquisadores têm se interessado em investigar se os lactentes são capazes de guiar seus movimentos baseados nessas informações. Dessa forma, o presente estudo tem por objetivo verificar se os lactentes tão jovens como os de 4 a 6 meses de vida são capazes de guiar seus movimentos realizando ajustes proximais e distais a partir das informações visual e tátil de tamanho e rigidez dos objetos.

Para responder o propósito deste estudo, duas hipóteses foram testadas. Embora Jeannerod ${ }^{9}$ afirme que as propriedades intrínsecas dos objetos, tal como o tamanho, afetam apenas os ajustes distais dos membros superiores, nossa primeira hipótese é a de que as propriedades intrínsecas, representadas neste estudo pelo tamanho (grande e pequeno) e rigidez (rígido e maleável) dos objetos, influenciarão tanto os ajustes proximais (alcance uni e bimanual) quanto os distais (mão aberta, semi-aberta, fechada, horizontal, vertical e oblíqua) do alcance manual. Isso porque acreditamos que, para alcançar e apreender com sucesso o objeto grande e rígido, o lactente precisará realizar ajuste bimanual, verticalização das mãos e mãos abertas; enquanto que, para alcançar e apreender o objeto grande e maleável, o lactente terá a opção de utilizar ajuste unimanual e, também, ajustes distais diversificados, sendo mesmo assim, capaz de apreender o objeto com sucesso. Quanto aos objetos pequenos, tanto o rígido quanto o maleável permitem a utilização de apenas uma mão, e os ajustes distais de orientação da mão poderão ser diversificados, sendo que a mão não precisa estar necessariamente aberta ao tocar os objetos para que estes sejam apreendidos.

Embora Corbetta, Thelen e Johnson ${ }^{10}$ e Fagard ${ }^{3}$ tenham verificado que os ajustes manuais ocorreram somente em lactentes por volta dos 8-9 meses de idade, nossa segunda hipótese é a de que os lactentes tão jovens quanto os de 4 a 6 meses apresentarão mudanças nos ajustes proximais e distais em função das variações de tamanho e rigidez dos objetos. Uma vez que os lactentes saudáveis iniciam os movimentos de alcance por volta dos 4 meses $^{11,14}$, e os de preensão voluntária, por volta dos 5 meses ${ }^{5,15,16,17,18}$, acreditase que a prática na execução dos movimentos dos braços ao longo dos 4 aos 6 meses de idade conduzirá ao refinamento das suas ações.

Portanto, acredita-se que os ajustes proximais e distais dos membros superiores dos lactentes serão influenciados pela percepção das propriedades físicas de tamanho e rigidez dos objetos, e bem como pela capacidade intrínseca do lactente 
de coordenar seus movimentos em uma complexa interação de percepção-ação. Obter maiores informações sobre os possíveis fatores que influenciam os movimentos de alcance e preensão de objetos parecem, portanto, ser de suma importância, visto que tais comportamentos são fundamentais para a continuidade do desenvolvimento motor e cognitivo do lactente.

\section{METODOLOGIA}

\section{Participantes}

Participaram do estudo nove lactentes saudáveis, três meninos e seis meninas, nascidos a termo ( $M=39$ semanas gestacionais; $\pm 1,41$ ). Esses foram avaliados longitudinalmente nas idades de $4(M=4$ meses e 2 dias; $\pm 0,85), 5(M=4$ meses e 26 dias; $\pm 1,32)$ e 6 ( $M=6$ meses e 1 dia; $\pm 2,55)$ meses, com tolerância de 5 dias anteriores ou posteriores à data do aniversário. O estudo foi aprovado pelo Comitê de Ética e Pesquisa com Seres Humanos (processo $n^{\circ}$ 040/30), e os pais ou responsáveis pelo lactente assinaram previamente o Termo de Consentimento Livre e Esclarecido.

\section{Materiais e Procedimentos}

Para a realização deste estudo foram utilizados quatro objetos esféricos e atrativos de propriedades distintas: dois maleáveis (“pompom” de lã antialérgica) e dois rígidos (bolas de isopor), sendo dois pequenos ( $5 \mathrm{~cm}$ de diâmetro) e dois grandes $(12,5 \mathrm{~cm} \text { de diâmetro })^{4,19,3,20}$. Em suma, foram apresentados aos lactentes um objeto rígido grande (RG), um rígido pequeno (RP), um maleável grande (MG) e um maleável pequeno (MP). Os objetos foram especialmente confeccionados para o propósito deste estudo.

Os lactentes foram posicionados em uma cadeira infantil ${ }^{21}$ com inclinação de $50^{\circ} \mathrm{com}$ a horizontal ${ }^{14}$. Um intervalo de 10 segundos foi permitido para que o lactente se adaptasse na postura e, então, com os lactentes em estado comportamental de alerta, iniciava-se o teste. Os objetos foram apresentados em diferentes seqüências pré-determinadas para que a ordem de apresentação não influenciasse nos resultados $^{22}$. Cada objeto foi apresentado a uma distância correspondente ao comprimento do membro superior do lactente, na linha média do corpo e na altura dos ombros ${ }^{10,16,17}$, por um período de 1 minuto ou até o lactente realizar 7 alcances. Um intervalo de 5 segundos foi permitido após cada apresentação, totalizando o tempo do procedimento em aproximadamente 4 minutos e 25 segundos.

Toda a fase experimental foi filmada por três câmeras filmadoras digitais, uma posicionada póstero-superiormente à cadeira e as outras duas localizadas anterior e diagonalmente à cadeira, estando uma à direita e a outra à esquerda ${ }^{21}$.

\section{Sistema de Análise}

As imagens foram capturadas por uma placa de captura de imagens, utilizando o software Adobe Premier 6.3 em arquivos com formato AVI. De posse desses arquivos, as imagens foram abertas no sistema Dvideow $5.0^{23}$, por meio do qual foi realizada a identificação, quadro a quadro, do início e final do alcance do membro superior que tocou o objeto, tanto das imagens referentes às câmeras situadas superior quanto lateralmente à cadeira. Para análise do movimento do membro superior esquerdo, por exemplo, analisamos as imagens das câmeras situadas superior e à esquerda da cadeira. Para os alcances bimanuais, foi analisado o membro superior que primeiro tocou o objeto.

\section{Descrição das variáveis dependentes}

\section{Ajustes proximais}

Foram considerados como ajustes proximais a iniciativa de direcionar um ou ambos os membros superiores ao alvo apresentado. Foi considerado ajuste unimanual quando o lactente deslocou somente um dos membros superiores em direção ao alvo ${ }^{10}$, ou quando ambos os membros saíram em direção ao objeto com uma diferença superior a 20 quadros (7,2 ms) do início do movimento de um membro para o outro $^{22}$, ou ainda, quando um dos membros superiores realizou o alcance do objeto enquanto o outro ficou parado ou produzindo pequenos movimentos que não fossem orientados ao objeto ${ }^{4}$. Foi considerado alcance bimanual quando o lactente estendeu simultaneamente os membros superiores em direção ao alvo $^{10}$, ou quando os membros superiores saíram da posição inicial com atraso igual ou inferior a 20 quadros de uma mão em relação à outra. Neste caso, as mãos deveriam deslocar simultaneamente até pelo menos a metade do arco de movimento (50\% da trajetória), sendo que o toque poderia ser feito simultaneamente com ambas as mãos, ou inicialmente com uma delas.

\section{Ajustes distais e Sucesso do alcance}

Foram considerados como ajustes distais aqueles realizados pelas mãos e dedos. Foi avaliada a orientação da mão, considerando as seguintes posições: horizontal - quando o antebraço estava em pronação, com a palma da mão voltada para baixo; vertical - quando o antebraço estava em posição neutra e a palma da mão orientada para a linha média do corpo do lactente; e oblíqua - quando a mão estava em posição intermediária em relação às outras duas supracitadas. A orientação da mão foi avaliada no momento em que o lactente tocou o objeto e quando realizou a preensão do mesmo. Referente à abertura da mão, foi considerada mão aberta quando as articulações metacarpofalangeanas e interfalangeanas estavam estendidas; mãos fechadas quando as articulações metacarpofalangeanas e interfalangeanas estavam fletidas; e mão semi-aberta quando as articulações metacarpofalangeanas estavam fletidas (independentemente do grau de flexão) e as interfalangeanas estendidas, ou, ainda, quando as metacarpofalangeanas estavam estendidas e as interfalangeanas 
fletidas ${ }^{22}$. A abertura da mão foi avaliada no início e no final do alcance.

Alcance com sucesso foi considerado quando o lactente conseguia apreender o objeto ou parte dele com uma ou ambas as mãos. Alcance sem sucesso foi determinado por movimentos direcionados ao objeto, seguido de toque; porém, que não resultassem em apreensão do mesmo ${ }^{22}$.

Para maior confiabilidade da análise dessas variáveis, foi realizado um Estudo de Fidedignidade interobservadores (3 observadores) e obtida concordância de 97,9\% entre eles.

\section{Análise dos Dados}

A análise dos dados foi concentrada nos movimentos de alcance, dos quais o início foi determinado como o primeiro movimento direcionado e ininterrupto do braço em direção ao objeto. $\mathrm{O}$ final do alcance foi determinado como o primeiro quadro no qual a mão contatou o objeto. Os alcances foram excluídos quando o lactente apresentava falta de interesse, choro ou irritação durante a realização do movimento ou quando iniciou o movimento com a mão próxima (trajetória inferior a 10 quadros) ao objeto.

Dos 7 possíveis alcances realizados para cada objeto, os primeiros 5 alcances foram utilizados para tratamento estatístico.

Nas análises dos movimentos de alcance, foi aplicada a técnica não paramétrica, visto que os dados são nominais. O Teste Qui-quadrado foi proposto para avaliar o percentual de alcances para os objetos RG, RP, MG e MP em todas as avaliações longitudinais.

Para todas as análises considerou-se um nível de significância de 0,05.

\section{RESULTADOS}

Um total de 432 alcances foi coletado, 12 foram excluídos por falta de interesse dos lactentes e 36 por erro no experimento (lactente iniciou o movimento com a mão próxima ao objeto). Assim, 384 alcances foram incluídos na análise.

Para verificar se a ordem de apresentação dos objetos não influenciou os resultados de ajustes proximais e distais de alcance, foi aplicado o Teste Kruskal Wallis, o qual constatou ausência de influência ( $p>0,05)$.

\section{Ajustes proximais em relação aos objetos apresentados}

Constatou-se diferença significativa nos ajustes proximais (uni e bimanual) em relação aos objetos apresentados $\left(x^{2}(3)=50,826 ; p<0,001\right)$. A Figura 1 ilustra maior porcentagem de ajustes bimanuais para o objeto RG e unimanuais para os demais objetos, principalmente para o RP.

\section{Ajustes distais em relação aos objetos apresentados \\ Constatou-se que não houve diferença significativa $\left(x^{2}\right.$ $(6)=3,189 ; p=0,785)$ na orientação da mão no toque em}

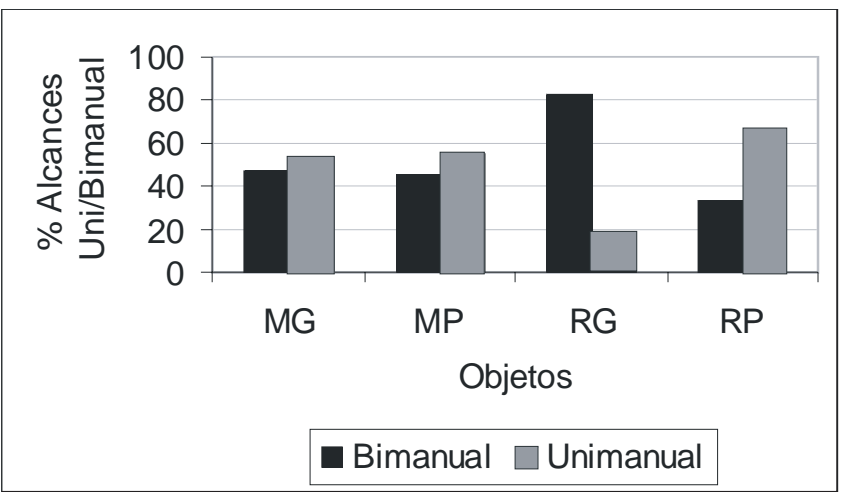

Figura 1. Percentual de alcances unimanual e bimanual para cada objeto apresentado: MG (maleável grande), MP (maleável pequeno), RG (rígido grande) e RP (rígido pequeno).

relação aos objetos apresentados (Figura 2A). Contudo, constatou-se diferença significativa da orientação da mão na preensão $\left(x^{2}(6)=17,876 ; p=0,007\right)$. A Figura 2 A mostra que a orientação da mão no toque dos objetos foi predominantemente oblíqua para todos os objetos. A Figura 2B mostra que a predominância de orientação da mão na preensão foi a vertical. O percentual de orientação da mão vertical, no entanto, foi maior para o objeto RG, e a orientação horizontal não foi observada na preensão deste objeto.
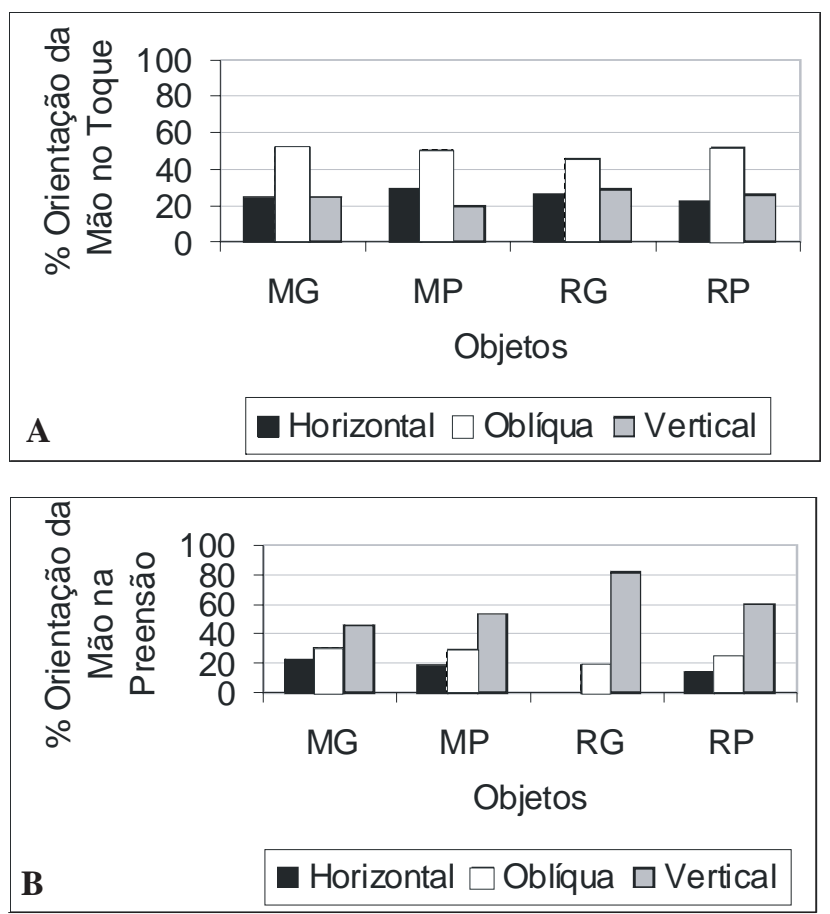

Figura 2. Percentual de orientação da mão para cada objeto apresentado: MG (maleável grande), MP (maleável pequeno), RG (rígido grande) e RP (rígido pequeno). A. Orientação da mão no toque dos objetos. B. Orientação da mão na preensão dos objetos. 
Constatou-se que não houve diferença significativa da abertura da mão no início do alcance $\left(x^{2}(3)=5,613 ; \mathrm{p}=0,468\right)$ em relação aos objetos apresentados (Figura 3A). No entanto, constatou-se diferença significativa na abertura da mão no final do alcance $\left(x^{2}(3)=9,551 ; p=0,023\right)$. A Figura 3A mostra que a abertura da mão no início do alcance foi predominantemente semi-aberta. A Figura 3B mostra que o percentual de mão aberta no final do alcance foi maior para o objeto RG quando comparado aos demais objetos.
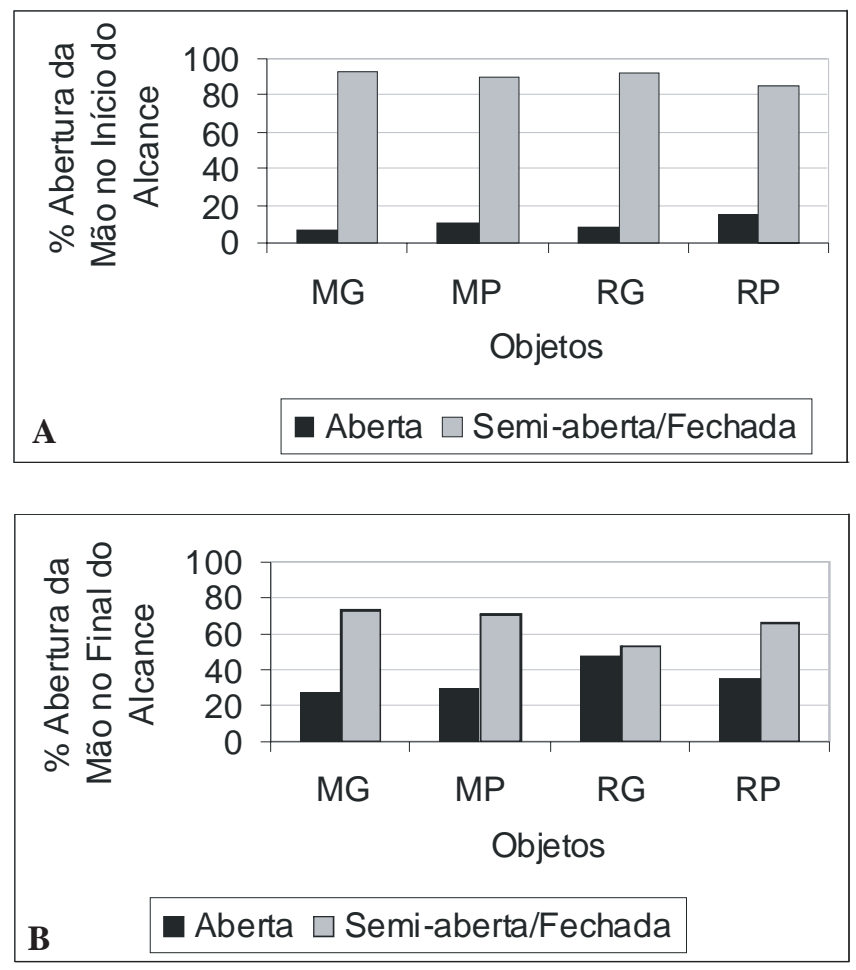

Figura 3. Percentual de abertura da mão para cada objeto apresentado: MG (maleável grande), MP (maleável pequeno), RG (rígido grande) e $\mathrm{RP}$ (rígido pequeno). A. Abertura da mão no início do alcance. B. Abertura da mão no final do alcance.

\section{Sucesso do alcance em relação aos objetos apresentados}

Constatou-se diferença significativa entre os alcances realizados com e sem sucesso em relação aos objetos apresentados $\left(x^{2}(3)=55,006 ; \mathrm{p}<0,001\right)$. A Figura 4 mostra que o percentual de alcances com sucesso foi maior para os objetos maleáveis (MP e MG) em relação aos rígidos (RP e $\mathrm{RG}$ ). Entre os objetos rígidos observa-se um percentual maior de alcances com sucesso para o objeto RP.

\section{DISCUSSÃO}

Influência das propriedades intrínsecas dos objetos nos ajustes proximais do alcance

O presente estudo disponibiliza evidências de que as propriedades de tamanho e rigidez dos objetos influenciam

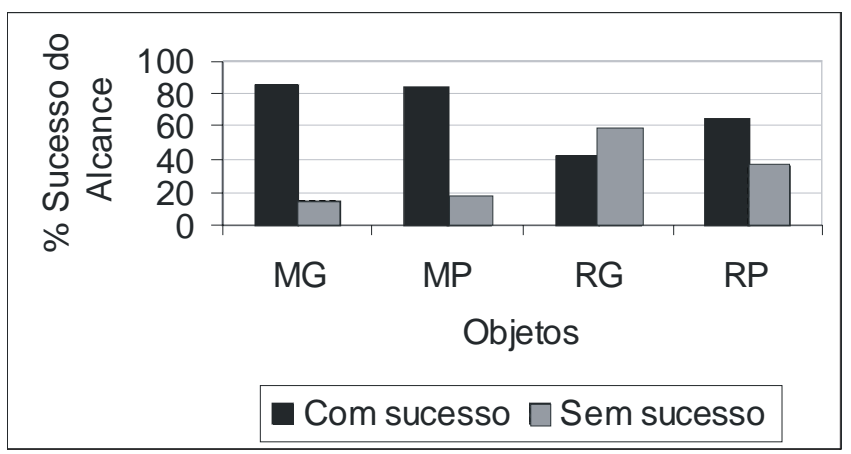

Figura 4. Percentual de alcances com e sem sucesso para cada objeto apresentado: MG (maleável grande), MP (maleável pequeno), RG (rígido grande) e RP (rígido pequeno).

tanto os ajustes proximais quanto os distais do alcance de lactentes tão jovens quanto os de quatro a seis meses de idade, confirmando, assim, as hipóteses previamente levantadas.

Verifica-se que os lactentes guiaram seus movimentos a partir da informação visual do tamanho e da rigidez dos objetos, realizando ajustes proximais bimanuais para o objeto RG e unimanuais para os demais objetos, principalmente para o RP, conforme esperado. Os resultados que indicam a influência do tamanho nos ajustes proximais estão de acordo com estudos de Newell et al., ${ }^{6,24}$. Estes verificaram que lactentes com 4-5 meses de idade foram capazes de usar uma mão para pegar um objeto pequeno e duas para pegar um objeto grande, sugerindo que o comportamento do lactente não é inflexível, mas, sim, funcionalmente adaptativo à restrição de tarefa. Entretanto, Fagard ${ }^{3}$, Corbetta, Thelen e Johnson ${ }^{10}$ verificaram que a capacidade do lactente em realizar ajustes proximais em relação ao tamanho ocorre somente por volta dos 8-9 meses. Fagard ${ }^{3}$ atribui tais resultados ao controle insuficiente principalmente de tronco superior, o qual seria necessário para manter a simetria corporal e permitir livre movimento de braço. No presente estudo, não considerouse que o insuficiente controle de tronco possa ter influenciado os ajustes dos movimentos dos lactentes, visto que a cadeira de teste permitiu o suporte de tronco apropriado, possibilitando o livre movimento de braços. Sendo assim, os resultados revelam que os lactentes jovens foram capazes de perceber as affordances dos objetos, bem como de gerar ações motoras apropriadas. Tal suposição reforça a idéia de Gibson ${ }^{13}$, que defende que a percepção guia a ação e a ação refina a percepção. Os resultados do presente estudo também estão de acordo com Oztop, Bradley e Arbib ${ }^{25}$ no qual verificaram por meio de modelo computacional que o contexto ambiental, bem como a repetição de tarefas modulam o desenvolvimento da preensão de lactentes jovens. Sugere-se, então, que a prática de movimentos diversificados durante dias, semanas e meses de vida dos lactentes estudados foi o que provavelmente conduziu ao refinamento das ações dos lactentes, e permitiu ajustes dos movimentos dos membros superiores. 
Referente à rigidez dos objetos, pode-se constatar que o objeto maleável ofereceu ao lactente a opção de usar apenas uma mão para alcançar e apreender os objetos independentemente de seu tamanho. Esse resultado está de acordo com o estudo de Corbetta, Thelen e Johnson ${ }^{10}$. Entretanto, neste estudo, os pesquisadores verificaram que tais ajustes ocorreram apenas em lactentes por volta dos 8 e 9 meses de idade, quando a restrição intrínseca foi reduzida. No presente estudo, acredita-se que os lactentes não apresentam restrição intrínseca que os impeçam de realizar ajustes proximais. Os lactentes tão jovens quanto os de 4 a 6 meses possuem padrões flexíveis de movimento que se adaptam às condições externas.

\section{Influência das propriedades intrínsecas dos objetos nos ajustes distais e sucesso do alcance}

Pode-se constatar que as propriedades intrínsecas dos objetos também influenciaram os ajustes distais do alcance no período estudado. Foi possível verificar que a orientação da mão no momento do toque foi predominantemente oblíqua, independentemente das propriedades dos objetos. Entretanto, para apreender os objetos, os lactentes precisaram mudar seus ajustes, passando a utilizar a orientação vertical, principalmente para o objeto RG, bem como, não utilizaram a orientação horizontal para este objeto. Portanto, nota-se que a orientação vertical parece ser a mais funcional para a preensão voluntária e a orientação horizontal parece não ser adequada para apreender o RG, pois exigiria do lactente disposição alternada dos membros superiores (uma mão sobre e acima e a outra, sob e abaixo do objeto) para manter o objeto RG nas mãos. Vale ressaltar que poucos estudos avaliaram o ajuste distal correspondente à orientação da mão, sendo que nenhum estudo foi identificado, avaliando ajustes entre o toque e a preensão dos objetos, bem como, utilizando objetos esféricos de diferentes tamanhos e rigidez. Von Hofsten e Fazel-Zandy ${ }^{26}$, ao avaliarem ajustes na orientação da mão utilizando como estímulos barras dispostas na vertical e na horizontal, também evidenciaram que lactentes tão jovens quanto os de 4 meses e meio de idade realizaram ajustes de orientação da mão em direção à orientação da barra antes mesmo de tocá-las.

Assim, tanto no estudo de Von Hofsten e Fazel-Zandy ${ }^{26}$ quanto neste estudo demonstrou-se que lactentes jovens mudaram suas estratégias para melhor ajustar a configuração da mão às propriedades e disposição dos objetos. Entretanto, no primeiro estudo, os lactentes ajustaram a palma das mãos antes do toque; enquanto neste, os lactentes ajustaram a orientação das mãos principalmente após o toque do objeto. Provavelmente, os lactentes precisaram da informação tátil adicional do tamanho e rigidez dos objetos, além das informações visual e proprioceptiva da relação do tamanho e orientação da mão com o objeto. Dessa forma, tais resultados avigoram o que Gibson ${ }^{13}$ preconiza sobre a informação que guia a ação, ou seja, a informação diz ao indivíduo o que deve ser feito e como deve ser feito. Segundo esse autor, o re- querimento de mais sistemas para levantar informações induz a descoberta de novas affordances. Os resultados do presente estudo revelam, portanto, que os lactentes usaram as informações visual, tátil e proprioceptiva para planejar e ajustar efetivamente seus movimentos.

Os lactentes ajustaram a abertura da mão em relação ao tamanho e rigidez dos objetos somente no final do movimento, o que foi observado pela predominância de mão aberta para o objeto RG, confirmando, assim, a hipótese levantada. Esse resultado está de acordo com Jakobson e Goodale $^{27}$, que demonstraram que a abertura da mão aumenta com o tamanho do objeto. Acredita-se que a mão deveria estar mais aberta para permitir a preensão do objeto grande e rígido, o que não seria necessário para apreender os objetos maleáveis, mesmo que grandes, pois a sua característica física facilita a entrada dos dedos dentro dos fios de lã, mesmo com as mãos semi-abertas. Essa mesma atribuição pode ser feita para o sucesso do alcance, visto que os objetos maleáveis eram mais facilmente apreendidos e mantidos nas mãos para serem explorados. Esses resultados estão de acordo com Corbetta, Thelen e Johnson $^{10}$, que afirmam que o objeto não tendo forma densa pode facilitar a preensão. Este resultado pode ser particularmente interessante no que se refere à intervenção fisioterapêutica, pois o comportamento de preensão de objetos é difícil de ser obtido em crianças com distúrbios neuromotores. Vale ressaltar que o objeto RG não foi o mais apropriado para estimular o comportamento de preensão nesta faixa etária, visto pela baixa porcentagem de alcances com sucesso. No entanto, este objeto parece ser apropriado para estimular comportamentos diversificados da mão tais como, ajustes de orientação vertical e maior abertura dos dedos.

Assim, esses achados sugerem mais uma vez que os lactentes jovens estudados perceberam as propriedades intrínsecas dos objetos e foram capazes de planejar e ajustar seus movimentos.

\section{CONSIDERAÇÕES FINAIS}

Este estudo mostra que os lactentes tão jovens quanto os de 4 a 6 meses de idade podem ser capazes de realizar ajustes proximais e distais em função do tamanho e rigidez dos objetos. Dessa forma, sugere-se que o desenvolvimento do alcance neste período de vida é marcado pela complexa interação de percepção-ação.

Apoio financeiro do CNPq.

\section{REFERÊNCIAS BIBLIOGRÁFICAS}

1. Thelen E, Corbetta D, Kamm K, Spencer J, Schneider K, Zernicke RF. The transition to reaching: Mapping intention and intrinsic dynamics. Child Dev 1993; 64: 1058-98. 
2. Corbetta D. Why do infants regress to two-handed reaching at the end of the $1^{\text {st }}$ year? [abstract] Infant Behav Dev 1998; 21: 42.

3. Fagard J. Linked proximal and distal changes in the reaching behavior of 5-to 12-month-old human infants grasping objects of different sizes. Infant Behav Dev 2000; 23: 317-329.

4. Corbetta D, Thelen E. The Developmental origins of bimanual coordination: a dynamic perspective. J Exp Psychol Hum Percept Perform 1996; 22: 502-522.

5. Hofsten $\mathrm{C}$ von, Rönnqvist L. Preparation for grasping an object: a developmental study. J Exp Psychol Hum Percept Perform 1988; 14(4): 610-621.

6. Newell KM, Scully DM, Mcdonald PV, Baillargeon R. Task constraints and infant grip configurations. Dev Psychobiol 1989; 22: 817-32.

7. Siddiqui A. Object size as a determinant of grasping in infancy. J Genet Psychol 1995; 153: 345-358.

8. Fagard J, Jacquet AY. Onset of bimanual coordination and symmetry versus asymmetry of movement. Infant Behav Dev 1989; 12: $229-235$.

9. Jeannerod M. Intersegmental coordination during reaching at natural visual objects. In: Long J, Baddeley A editores. Attention and Performance IX. Hillsdale: Erlbaum; 1981. P. 153-168.

10. Corbetta D, Thelen E, Johnson K. Motor constraints on the development of perception-action matching in infant reaching. Infant Behav Dev 2000; 23: 351-74.

11. Thelen E, Corbetta D, Spencer JP. Development of reaching during the first year: Role of movement speed. J Exp Psychol Hum Percept Perform 1996; 22: 1059-76.

12. Gibson JJ. The ecological approach to visual perception. Hillsdale (NJ): Laurence Erlbaum Associates; 1986. P. 322.

13. Gibson EJ. Exploratory behavior in the development of perceiving, acting, and the acquiring of knowledge. In: Advances in infancy research, Norwood (NJ.): Ablex Publishing Company; 1995. P. 21-61.

14. Hofsten $C$ von. Developmental changes in the organization of prereaching movements. Dev Psychol 1984; 20: 378-386.

15. Savelsbergh GJP, Kamp van der J. The effect of body orientation to gravity on early infant reaching. J Exp Child Psychol 1994; 58: 510-528.
16. Thelen E, Spencer JP. Postural control during reaching in young infants: a dynamic systems approach. Neurosci Biobehav Rev 1998; 22(4): 507-514.

17. Fits IBM van der, Hadders-Algra M. The development of postural response patterns during reaching in healthy infants. Neurosci Biobehav Rev 1998; 22(4): 521-526.

18. Newman C, Atkinson J, Braddick O. The developmental of reaching and looking preferences in infants to objects of different sizes. Dev Psychol 2001; 37(4): 561-572.

19. Fagard J, Pezé A. Age changes in interlimb coupling and the development of bimanual coordination. J Mot Behav 1997; 29(3): 199-208.

20. Hof P van, Kamp J van der, Savelsbergh GJP. The relation of unimanual and bimanual reaching to crossing the midline. Child Dev 2002; 73: 1353-63.

21. Carvalho RP, Tudella E, Barros RML. Utilização do Sistema Dvideow na análise cinemática do alcance manual de lactentes. Rev. Bras Fisioter 2005; 9(1): 1-7.

22. Rocha NACF. Impacto das propriedades físicas dos objetos nos movimentos de alcance manual em lactentes saudáveis de 4 a 6 meses de idade [Tese]. São Carlos (SP): Universidade Federal de São Carlos, 2006. Disponível em http://www.bdtd.ufscar.br/ tde_busca/arquivo.php?codArquivo=940.

23. Figueroa PJ, Leite NJ, Barros RML. A flexible software for tracking of markers used in human motion analysis. Comput Methods Programs Biomed 2003; 72: 155-165.

24. Newell KM, McDonald PV, Baillargeon R. Body scale and infant grip configurations. Dev Psychobiol 1993; 26(4): 195-205.

25. Oztop E, Bradley NS, Arbib MA. Infant grasp learning: a computational model. Exp Brain Res 2004; 158(4): 480-503.

26. Hofsten C von, Fazel-Zandy S. Development of visually guided hand orientation in reaching. J Exp Child Psychol 1984; 38: 208219.

27. Jakobson LS, Goodale MA. Factors affecting higher-order movement planning: a kinematic analysis of human prehension. Exp Brain Res 1991; 86: 199-208. 